Frequent SMU enabled young people to maintain and expand their social networks but a need to be constantly available was sometimes overwhelming, suggesting an 'over-stimulation' effect.

Conclusion Caregivers and teachers should take a nuanced approach to addressing young people's SMU rather than following the dominant alarmist discourse. A measured approach should be taken, providing clear, reasonable guidance and boundary-setting but also promoting trust and responsible time management, and acknowledging the role of social media in making connections. Understanding and sharing in online experiences is likely to promote social connectedness. Supporting young people to negotiate breathing space in online interactions and prioritising trust over availability in peer relationships may optimise the role of social media in promoting peer connectedness in particular.

\section{P09 ESTIMATION OF THE CAUSAL EFFECT OF CHURCH ATTENDANCE ON RISK OF MYCOBACTERIUM TUBERCULOSIS INFECTION IN YOUNG CHILDREN IN RURAL MALAWI USING TARGETED MAXIMUM LIKELIHOOD ESTIMATION}

\begin{abstract}
${ }^{1}$ Palwasha Khan*, ${ }^{2}$ Kathy Baisley, ${ }^{3}$ Leo Martinez, ${ }^{4}$ Themba Mzembe, ${ }^{4}$ Regina Chiumya,

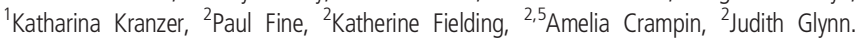
${ }^{1}$ Clinical Research, London School of Hygiene and Tropical Medicine, London, UK; ${ }^{2}$ Infectious Disease Epidemiology, London School of Hygiene and Tropical Medicine, London, UK; ${ }^{3}$ Epidemiology, Boston University, School of Public Health, Boston, USA; ${ }^{4}$ Karonga site, Malawi Epidemiology and Intervention Research Unit, Karonga, Malawi; ${ }^{5}$ Lilongwe site, Malawi Epidemiology and Intervention Research Unit, Lilongwe, Malawi
\end{abstract}

\subsection{6/jech-2021-SSMabstracts.99}

Background Important gaps exist in our understanding of Mycobacterium tuberculosis transmission, especially in high HIV prevalence settings. There is significant uncertainty about where most transmission takes place in the community, impeding control efforts. M. tuberculosis infection in young children is a sensitive indicator of recent transmission and may provide a means of identifying locales of community transmission. We estimate the causal effect of church attendance on incident $M$. tuberculosis infection in young children.

Methods Children aged under six years residing within a demographic surveillance site in Malawi were recruited. Tuberculin skin testing (TST) was performed at baseline and repeated after 1-2 years. At the time of the 2 nd TST, structured guardian interviews were undertaken to ascertain any known contact with tuberculosis within the previous 12-18 months. Detailed household socioeconomic and demographic data were available. Very few children had never attended church in the previous year $(<4 \%)$ so the unexposed group was combined with the next category (1-3 attendances) to form the baseline. Incident infection was defined as an increase in TST induration of $\geq 13 \mathrm{~mm}$ from 1 st TST to $2 \mathrm{nd}$ TST among those with indurations of $<10 \mathrm{~mm}$ at baseline (based on mixture analysis). We used hierarchical targeted maximum likelihood estimation (TMLE) to estimate the causal effect of church attendance on infection incidence accounting for clustering at the community-level. Confounders included age, sex, household socioeconomic status and time between TSTs.

Results 2349 children were eligible for inclusion, of whom 2019 (86\%) had a 2nd TST placed and data on church attendance. 66 (3.2\%: 95\% CI 2.5-4.1) children had evidence of TST-conversion; 3/1795 (1.4\%: 95\% CI 0.3 - 4.0) among those with $<4$ attendances and 633.3\%: 95\% CI 2.5-4.2) in those with $\geq 4$ attendances. The estimated adjusted risk ratio was 2.8 (95\%CI 1.2 - 6.7; p-value 0.023$)$ for church attendance compared to baseline group.

Conclusion High levels of church attendance ( $\geq 4$ per year) increased the risk of incident infection by 2.8 -fold compared to church attendance of less than 4 attendances per year in young children in rural Malawi. Simple infection control practices, such as opening windows or even holding congregations in outdoor spaces, may be highly beneficial in mitigating the risk of community $M$. tuberculosis transmission and other respiratory/airborne pathogens.

\section{P10 REALIST REVIEW LINKING POLITICAL EXPOSURES TO CHILD AND MATERNAL HEALTH OUTCOMES}

${ }^{1}$ Maxwell Barnish*, ${ }^{2}$ Si Ying Tan, ${ }^{2}$ Araz Taeihagh, ${ }^{3}$ Michelle Tørnes, ${ }^{4}$ Rebecca NelsonHorne, ${ }^{1}$ GJ Melendez-Torres. ${ }^{1}$ Peninsula Technology Assessment Group (PenTAG), University of Exeter Medical School, Exeter, UK; ${ }^{2}$ Policy Systems Group (PSG), Lee Kuan Yew School of Public Policy, National University of Singapore, Singapore; ${ }^{3}$ Ageing, Clinical and Experimental Research (ACER) Team, Institute of Applied Health Sciences, University of Aberdeen, Aberdeen, UK; ${ }^{4}$ Independent Scholar, Glasgow, UK

\subsection{6/jech-2021-SSMabstracts. 100}

Background There are longstanding conceptual and theoretical links between political exposures and population health. Systematic reviews have demonstrated that the welfare state, political tradition, democracy and globalisation exert an important influence on population health outcomes. However, there has been no systematic analysis of the mechanisms by which these effects may operate. Therefore, taking a focus on child and maternal health outcomes, a realist re-synthesis of the dataset from an existing systematic review is presented.

Methods In order to systematically evaluate the mechanisms by which political effects on child and maternal health operate using realist methods, searches from an existing systematic review up to November 2017 were used. Ten databases were searched, and supplementary web searches and citation chasing were conducted. Included studies quantitatively investigated the link between the welfare state, political tradition, democracy or globalisation and child or maternal health outcomes in at least two countries. Following standardised duplicate screening and data extraction, initial realist theory generation took place, followed by theory adjudication to determine final theories. As realist methods were used, there was no standardised assessment of risk of bias.

Results 35,333 unique records were identified, of which 255 proceeded to full-text review, 176 to inclusion in the original systematic review, and 67 were included in this realist review on child and maternal health outcomes. Sixty-three of these studies were ecological and included data from 1950-2014. Six initial theories were generated. Following theory adjudication, three theories in revised form were supported and formed the final programme theories. These related to a more generous welfare state leading to better child and maternal health especially in developed countries through progressive social welfare policies, left-of-centre political tradition leading to lower child mortality and low birth weight especially in developed countries through greater focus on welfare measures, and increased globalisation leading to greater child and infant mortality and youth smoking rates in LMECs through 
greater influence of multinational corporations and neoliberal trade organisations.

Conclusion Following a realist re-synthesis of a large systematically collected dataset, three final programme theories were supported that offer an account of how the welfare state, political tradition and globalisation may exert an important effect on child and maternal health. Limitations include lack of stratification by level of development, contextual effects in interpreting fertility rates, and complexity in mapping political exposures to political parties internationally. Future realist analyses could consider other political exposures, such as governance and political capacity.

\section{P11 HOW DO PERINATAL RISK FACTORS MEDIATE THE RELATIONSHIP BETWEEN MATERNAL SOCIOECONOMIC STATUS AND PRETERM BIRTH? ANALYSIS OF ROUTINE HEALTHCARE DATA FROM THE NORTH WEST REGION OF ENGLAND}

${ }^{1}$ Philip McHale*, ${ }^{1}$ Daniela Schlüter, ${ }^{1}$ Ben Barr, ${ }^{2}$ Shantini Paranjothy, ${ }^{3}$ Angharad Care, ${ }^{1}$ David Taylor-Robinson. 'Department of Public Health, Policy and Systems, University of Liverpool, Liverpool, UK; ${ }^{2}$ Aberdeen Health Data Science Research Centre, University of Aberdeen, Aberdeen, UK; ${ }^{3}$ Centre for Women's Health Research, University of Liverpool, Liverpool, UK

\subsection{6/jech-2021-SSMabstracts. 101}

Background Prevalence of preterm birth (PTB) is increasing in England. Given significant inequalities in prevalence by maternal socioeconomic status (SES) and subsequent negative consequences on health and educational attainment for children, this represents a significant risk of widening population health inequalities. The aim is to understand how different exposures to risks mediate the relationship between SES and PTB.

Methods Analysis of routinely collected data for all births at Liverpool Women's Hospital between April 2009 and March 2020, covering an area of North West England with high levels of deprivation. The exposure was a dichotomised measure of maternal SES at birth (two most deprived Indices of Multiple Deprivation deciles compared to other eight deciles), and the outcome was PTB (gestational age $<37$ weeks) compared with term. Mediators were maternal smoking status, medical conditions, obstetric conditions, and BMI at booking. Covariates included maternal age at birth, method of labour onset, parity, baby sex, and previous PTB. Mediation was assessed using multivariable logistic regression for modelling the effect of SES on PTB after adjusting for covariates (minimally adjusted) with and without adjustment for the potential mediators and applying difference-of-coefficients method; the difference in odds ratio (OR) for SES between these models. Proportion mediated is $\left(\mathrm{OR}_{\text {mediator-adjusted - }}\right.$ $\left.\mathrm{OR}_{\text {minimally-adjusted }}\right) /\left(\mathrm{OR}_{\text {minimally-adjusted }}-1\right)$. All analysis was done in $\mathrm{R}$.

Results Analysis sample consisted of 81,680 births; $7.4 \%$ were preterm, while $64.1 \%$ were to low SES mothers. Of mothers, $19.1 \%$ were current smokers, $4.3 \%$ had medical conditions, $10.1 \%$ had obstetric conditions and $48.6 \%$ had healthy weight BMI. The OR for the effect of low SES on PTB in the minimally adjusted model was 1.26 (95\% CI 1.19-1.34). When mediators were included, OR reduced by $42 \%$ for smoking. OR for low SES did not change when maternal medical conditions or obstetric conditions were included, while OR increased when BMI was included (1.32, 95\% CI 1.22-1.44).

Discussion Our findings demonstrate that significant inequalities exist in the prevalence of PTB. The initial results from the mediation analysis suggest that smoking is partly explains these inequalities. Over half of this inequality is not explained by mediators included here. Limitations include unavailable data on other mediators (e.g. maternal mental health) and in the analysis approach. The next step is to analyse the data using the counterfactual approach to mediation. Reducing PTB is a priority for Maternity and Children's services, and the NHS' Long Term Plan. These results suggest that action on smoking during pregnancy will reduce inequalities.

\section{P12 INVESTIGATING CAUSALITY BETWEEN ADIPOSITY AND WOMEN'S REPRODUCTIVE FACTORS: A MENDELIAN RANDOMIZATION ANALYSIS}

Claire Prince*, Gemma C Sharp, Laura D Howe, Abigail Fraser, Rebecca C Richmond. MRC Integrative Epidemiology Unit, Bristol Medical School, University of Bristol, Bristol, UK

\subsection{6/jech-2021-SSMabstracts. 102}

Background Reproductive factors (RFs) in women, such as menarche and menopause, the age at which women start and stop having children, and the number of children they have, have been implicating in disease risk including, breast cancer, cardiovascular, respiratory diseases. While body mass index (BMI) has similarly been identified as a risk factor in such diseases. Studies have identified strong bidirectional associations between adulthood BMI and RFs in women, and higher body size in girls was reported to be associated with earlier age at menarche and menopause. Considering the evidence of interplay between BMI and RFs, it is important to consider causal relationships which may underlie these associations. This study aims to appraise causality between both childhood and adulthood adiposity and the RFs, as well as establishing the magnitude of any identified relationships and direction of causality.

Methods We used data from the UK Biobank and genetic consortia with data available for RFs and BMI. We then applied Mendelian randomization (MR) methods to estimate the causal relationships between RFs and BMI both in childhood and adulthood. Sensitivity analyses were used to investigate directionality of the effect observed, to test for evidence of pleiotropy and to account for any sample overlap.

Results MR revealed body size and BMI in childhood and adulthood respectively have potentially causal relationships with a number of RFs, as well as a number of reproductive having a potentially effect on BMI in adulthood. Notably higher body size during childhood appears to lead to an earlier age at menarche $(\operatorname{Beta}(\mathrm{B})=-0.65,95 \%$ confidence intervals $(\mathrm{CI})=-0.74,-0.56)$, age at first, $(B=-0.07, C I=-0.12$, $0.01)$ and last birth $(B=-0.09, C I=-0.13,-0.04)$. While higher BMI during adulthood seems to lead to an earlier age at menopause $(B=-0.53, C I=-0.82,-0.23)$, age at first live birth $(B=-$ $1.72, \mathrm{CI}=-2.06,-1.39)$, age at last live birth $(\mathrm{B}=-1.40, \mathrm{CI}=-$ $1.68,-1.13)$, earlier age at first sexual intercourse $(B=-1.09$, $\mathrm{CI}=-1.39,-0.78)$ and increased likelihood of having children $(\mathrm{OR}=1.11, \mathrm{CI}=1.01,1.21)$. Finally, findings suggests that earlier menarche $(B=-0.03, C I=-0.04,-0.03)$ and age at first birth $(B=-0.05, C I=-0.06,-0.03)$ and age first had sexual 